\title{
Neutrino Physics at Fermilab in the 1990s
}

\author{
R.H. Bernstein \\ Fermi National Accelerator Laboratory \\ P.O. Box 500, Batavia, Illinois 60510
}

October 1992

Presented at Neutrino '92,

Granada, Spain, June 7-12, 1992 


\section{Disclaimer}

This report was prepared as an account of work sponsored by an agency of the United States Government. Neither the United States Government nor any agency thereof, nor any of their employees, makes any warranty, express or implied, or assumes any legal liability or responsibility for the accuracy, completeness, or usefulness of any information, apparatus, product, or process disclosed, or represents that its use would not infringe privately owned rights. Reference herein to any specific commercial product, process, or service by trade name, trademark, manufacturer, or otherwise, does not necessarily constitute or imply its endorsement, recommendation, or favoring by the United States Government or any agency thereof. The views and opinions of authors expressed herein do not necessarily state or reflect those of the United States Government or any agency thereof. 


\title{
Neutrino Physics at Fermilab in the 1990's
}

\author{
R.H. Bernstein \\ a Fermi National Accelerator Laboratory, Batavia IL 60510, USA
}

Three sets of proposed neutrino experiments at Fermilab are discussed. The first experiment (E-815) is a continuation of the deep-inelastic scattering program at the Tevatron, performing precise measurements of the weak mixing angle and structure functions. The second $(P-860)$ is a proposal to use the Fermilab Debuncher to search for neutrino oscillations. The third is a suggestion for a tagged neutrino beam which could be of use in measuring neutrino oscillation parameters.

\section{Introduction}

I will discuss three experiments which have been proposed at Fermilab. The first, a continuation of the successful deep-inelastic neutrino scattering program, has been approved for the $\mathbf{1 9 9 4}$ fixed-target run. The second is an active proposal to use the Fermilab Debuncher in a search for neutrino oscillations. Finally, with the intensity upgrades from the Main Injector, it is possible to construct the first tagged neutrino beam and measure the oscillation parameters $\Delta m^{2}$ and $\sin ^{2} 2 \theta$. I do not cover the short- and longbaseline oscillation experiments since they are covered separately in this Conference.[1]

\section{Deep-Inelastic Scattering}

\subsection{Physics Goals of $\mathbf{E}-\mathbf{8 1 5}$}

E-815 was approved in Summer 1992 to measure the weak mixing angle and $\rho$ in a new, precision experiment at the Fermilab Tevatron. It will use the 690 Ton Fe-target CCFR detector[2] (with small modifications) and a new, sign-selected quadrupole triplet beam to measure $R_{\nu}$ and $R_{\nu}$. The sign-selection, together with the known $r=\sigma(\bar{\nu}, \mathrm{CC}) / \sigma(\nu, \mathrm{CC})$ will make $\mathrm{E}-$ 815 the first experiment to measure the PaschosWolfenstein variables $R^{+}$and $R^{-}$. Improvements in the beam, along with experience in E-744 and E-770, will enable the experiment to significantly improve on existing errors, and provide the first precision measurement of $\rho$.[3]

\subsubsection{Precision Measurement of $\sin ^{2} \theta_{W}$}

The recent improvements in direct measurements of $M_{W}$ and $M_{Z}$ demand a new measurement of $\sin ^{2} \theta_{W}$ in deep-inelastic scattering. Comparisons of $\sin ^{2} \theta_{W}$ in DIS to measurements in other processes are probes of the radiative corrections to the Standard Model. The most important example is the upper limit on $m_{t}$, extracted by comparing $\sin ^{2} \theta_{W}$ measured in DIS to the Sirlin variable $\sin ^{2} \theta_{W}=1-M_{W}^{2} / M_{Z}^{2} \cdot$.[4] One way to make the comparison is to work within the Standard Model and convert all measurements to an equivalent $M_{W}$ (using the measured $M_{Z}$ as a fundamental constant), shown in Table 1 . We then see that over the next decade, deep-inelastic scattering can keep pace with collider measurements, shown below (we assume $M_{Z}=91.175 \pm 0.021$ $\mathrm{GeV}$ ).

2.1.2. The fixst precise determination of $\rho$ There are two parameters in the theory: $\sin ^{2} \theta_{W}$ and $\rho$, or conversely $S$ and $T . \rho$ is sensitive to $m_{t}$ and isospin-breaking effects. It is also a probe of the Higgs sector. The Veltman definition of $\rho$ shows that it is a function of the VEV of the Higgs fields: for Standard Model doublets, $\rho=1$ at tree-level; if Higgs occur in triplets, then $\rho$ will differ from unity.[5]

The value of $\rho$ is also a quadratic function of $m_{t}$ :

$\Delta \rho=\frac{3 \alpha}{16 \pi \sin ^{2} \theta_{W}}\left(\frac{m_{t}}{M_{W}}\right)^{2}$

and so a precise value of $\rho$ may be used to either (1) predict $m_{t}$, or (2) check for consistency with a measured $m_{t}$. Eventually this experiment will 
Present Situation

\begin{tabular}{|l|c|}
\hline \multicolumn{1}{|c|}{ Measurement } & Equivalent W Mass \\
\hline CDF/UA2 $M_{W}$ & $80.14 \pm 0.27 \mathrm{GeV}$ \\
Neutrino DIS & $79.90 \pm 0.30 \mathrm{GeV}$ \\
& \\
LEP (700K Events) & $80.01+0.27 /-0.37 \mathrm{GeV}$ \\
\hline
\end{tabular}

Future (3-5 Years)

\begin{tabular}{|l|l|}
\hline \multicolumn{1}{|c|}{ Measurement } & Future Error \\
\hline CDF/D0 Mass & $\pm 0.100 \mathrm{GeV}$ \\
E-815 Neutrino & $\pm 0.100 \mathrm{GeV}$ \\
& \\
LEP II & $\pm 0.100 \mathrm{GeV}$ \\
\hline
\end{tabular}

Post Main Injector

\begin{tabular}{|l|c|}
\hline Measurement & Future Error \\
\hline CDF/D0 Mass & $\pm 0.050 \mathrm{GeV}$ \\
& \\
E-815 Neutrino & $\pm 0.060 \mathrm{GeV}$ \\
\hline
\end{tabular}

Table 1

The evolution of the relevant experiments throughout the 1990's, expressed as measurements of $M_{W}$. It is seen that DIS keeps pace with the collider measurements as a probe of the radiative corrections to the Standard Model.

be able to check $m_{t}$ with errors of approximately $\pm 10 \mathrm{GeV} / \mathrm{c}^{2}$.

We recall that in the Llewellyn Smith formulation, $[6]$

$$
\begin{aligned}
R_{\nu} & =\frac{\sigma\left(\nu_{\mu}, \mathrm{NC}\right)}{\sigma\left(\nu_{\mu}, \mathrm{CC}\right)} \\
& \approx \rho^{2}\left(\frac{1}{2}-\sin ^{2} \theta_{W}+\frac{5}{9} \sin ^{4} \theta_{W}(1+r)\right) \\
R_{D} & =\frac{\sigma\left(\bar{\nu}_{\mu}, \mathrm{NC}\right)}{\sigma\left(\bar{\nu}_{\mu}, \mathrm{CC}\right)} \\
& \approx \rho^{2}\left(\frac{1}{2}-\sin ^{2} \theta_{W}+\frac{5}{9} \sin ^{4} \theta_{W}\left(1+\frac{1}{r}\right)\right)
\end{aligned}
$$

where $r=\sigma(\bar{\nu}, \mathrm{CC}) / \sigma(\nu, \mathrm{CC})$, and so there are two equations and two unknowns. The Paschos-
Wolfenstein variables are:[7]

$$
\begin{aligned}
R^{-} & =\frac{\sigma\left(\nu_{\mu}, N C\right)-\sigma\left(\bar{\nu}_{\mu}, N C\right)}{\sigma\left(\nu_{\mu}, C C\right)-\sigma\left(\bar{\nu}_{\mu}, C C\right)} \\
& \approx \rho^{2}\left(\frac{1}{2}-\sin ^{2} \theta_{W}\right) \\
R^{+} & =\frac{\sigma\left(\nu_{\mu}, N C\right)+\sigma\left(\bar{\nu}_{\mu}, N C\right)}{\sigma\left(\nu_{\mu}, C C\right)+\sigma\left(\bar{\nu}_{\mu}, C C\right)} \\
& \approx \rho^{2}\left(\frac{1}{2}-\sin ^{2} \theta+\frac{10}{9} \sin ^{4} \theta_{W}\right)
\end{aligned}
$$

and

$$
R^{ \pm}=\frac{R_{\nu} \pm R_{D}}{1 \pm r}
$$

The fact that $R^{ \pm}$are linear combinations of $R_{\nu, D}$ implies that if we know $r$, then we can obtain $R^{ \pm}$from the measured ratios. Knowledge of $r$ is equivalent to having measured the structure functions, and that data has been obtained from E744/E-770 in the CCFR detector at similar energies.[11] Hence no absolute flux measurement is necessary to use the Paschos-Wolfenstein technique.

With the E-815 Sign-Selected Beam, we will have both $\nu$ and $\bar{\nu}$ data enabling us to combine the results and measure both $\rho$ and $\sin ^{2} \theta_{W}$. We will measure $\rho$ to better than $1 \%$, a factor of three better than earlier measurements.[10]

A combined fit to $\rho$ and $\sin ^{2} \theta_{W}$ yields an ellipse; we compare the deep-inelastic result to that obtainable from $M_{Z}$ and $M_{W}$ in Fig. 1. Note that the ellipse is nearly orthogonal to the $M_{Z}$ band, maximizing the power of the test. The ellipse and the $M_{W}$ stripe are almost the same width, indicating the relative strengths in $\sin ^{2} \theta_{W}$ are about equal; $M_{W}$, on the other hand, gives no information about $\rho$.

\subsection{Other Standard Model Tests}

Precise determinations of $\rho$ and $\sin ^{2} \theta_{W}$ also can be used to study a number of other extensions to the Standard Model. Langacker, Luo, and Mann[8] have detailed some of these tests: a non-standard Higgs, sector, new heavy quarks, or extra $Z$-bosons are just three tests in which deepinelastic scattering can provide uniquely precise data. Interpreting $\rho$ and $\sin ^{2} \theta_{W}$ in the $S, T$ framework provides a powerful test of technicolor 


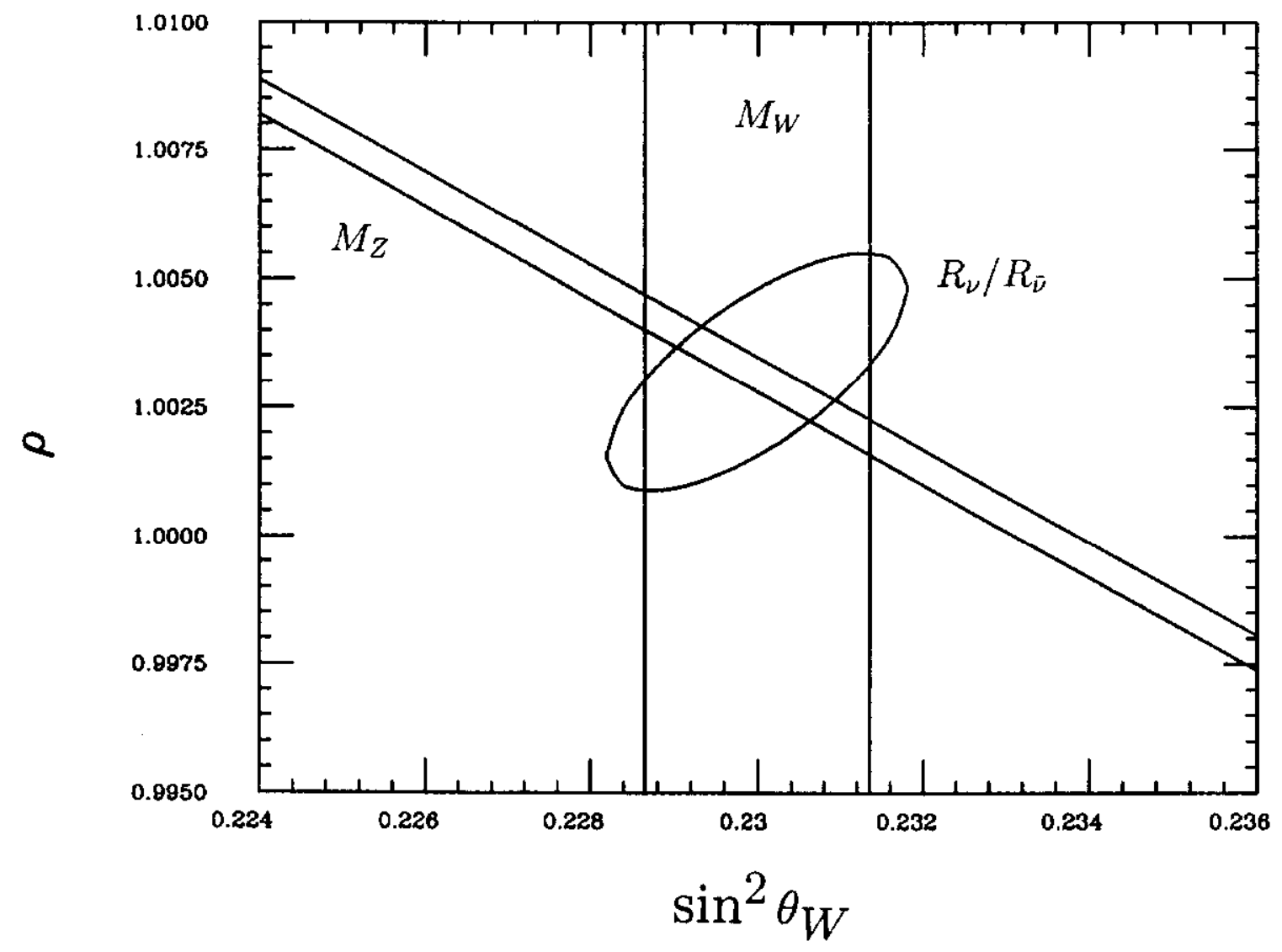

Figure 1. Combined Measurement of $\rho$ and $\sin ^{2} \theta_{w}$ in a post-Main Injector experiment. The Standard Model with $m_{t}=150 \mathrm{GeV} / \mathrm{c}^{2}$ is assumed.

a well, clearly identifying a single tecnhicolor generation.

\subsection{Sign-Selected Quadrupole Triplet}

The Quadrupole Triplet Beam used in E744/E-770 focuses positively and negatively charged mesons and allows neutrals to pass through undeflected. The sign-selection embeds the Quadrupole Triplet (with some improvements over the old system) in a pair of dipoles. The first dipole immediately follows the target and bends the desired polarity into the Quadrupole Triplet channel. The train is set for a point-toparallel focus at $300 \mathrm{GeV} / \mathrm{c}$. Following the train is a second dipole which bends the resultant beam toward the detector. The two bends are necessary to minimise backgrounds from decays within the train. Although the entire system works in the $x-z$ plane (instead of a three-dimentsional corkscrew) the resultant dispersion and variation of momentum across the face of the detector are acceptably small.

\subsection{Sources of Error}

Let us explore the sources of exror in more detail. The current world average on $\sin ^{2} \theta_{w}$ is $0.234 \pm 0.003 \pm 0.004$, where the second error is dominated by the systematic errors associated with alow-rescaling. Slow-rescaling parameterises the effect of the heavy charm quark on the crosesection through a parameter $m_{e}$, determined from opposite-sign dimnon production.[9] E-815 can largely eliminate this sonrce of error by using the Pachos-Wolfenstein $R^{-}$; the expected precision is \pm 0.0024 from Tevatron runs before the Main Injector. The statistical error of the experiment will be \pm 0.011 , and many of the remaining systematics will be dominated by the statistics of the event sample. The use of $R^{-}$will reduce the error from slow-rescaling to only \pm 0.0005 . Such a technique, however, requires setting $\rho$ to the Standard Model value; in the formalism, that is equivalent to using one of the equations (for $R_{D}$ or $R^{+}$) to set $m_{c}$ instead of measuring $\rho$.

Another major source of errot is the $\nu_{e}$ flux. E-744/E-770 used a Quadrupole Triplet beam 


\begin{tabular}{|l|c||c|}
\hline Species & CC Events & NC After All Cuts \\
\hline \multicolumn{3}{|c|}{ Neutrino Mode } \\
\hline$\nu_{\mu}$ & $2400 K$ & $52 / K$ \\
$\bar{\nu}_{\mu}$ & 540 & 200 \\
$\nu_{e}\left(K_{L}\right)$ & 1400 & 476 \\
\hline \multicolumn{3}{|c|}{ Antineutrino Mode } \\
\hline$\nu_{\mu}$ & 1300 & 400 \\
$\bar{\nu}_{\mu}$ & $450 K$ & $72 K$ \\
$\nu_{e}\left(K_{L}\right)$ & 1600 & 545 \\
\hline
\end{tabular}

Table 2

Event Rates for E815 for $2 \times 10^{18}$ protons-ontarget in the Sign-Selected Beam. The runs have been equally divided between neutrino and antineutrino modes. The mean neutrino energy is $147 \mathrm{GeV}$; for antineutrinos, it is $124 \mathrm{GeV}$. The desired channel is italicized. The background neutrinos have mean energies of approximately 50 GeV.

which permitted electron neutrinos from $K_{L} \rightarrow$ $\pi e \nu_{e}$ to hit the detector. The CCFR detector is not suitably instrumented to cleanly separate charged-current $\nu_{a}$ interactions from $\nu_{\mu}$ neutral current events. The electron is lost in the resulting hadronic shower and hence all $\nu_{e}$ look like neutral currents. The $\nu_{e}$ from $K_{L}$ decay are difficult to model since the $K_{L}$ production rate is not known to better than $20 \%$, yielding an error of $\approx \pm 0.003$ in $\sin ^{2} \theta_{W}$. This is unacceptably large for a precision measurement. E-815's signselected quadrupole triplet will eliminate the $K_{L}$ from the beam: the desired polarity will be bent toward the detector, causing the unwanted particles to either strike collimators or point them away from the apparatus. $\nu_{e}$ from $K^{ \pm}$can be extracted from the data by measuring the flux from $K^{ \pm} \rightarrow \pi^{\circ} \mu \nu_{\mu}$ which make up the high-energy end of the charged-current data.

Rates and backgrounds for both $\nu_{\mu}$ and $\vec{\nu}_{\mu}$ runs are shown in Table 2.

Other sources of error in the CCFR apparatus at Tevatron energies include the structure functions, hadron energy definition, and the neutral/charged current separation algorithm. These have been studied in the $\mathrm{E}-744 / \mathrm{E}-770$ analy-

\begin{tabular}{|l|c|c|}
\hline Contribution & E-770 & E-815 \\
\hline Statistics & 0.0023 & 0.0011 \\
\hline Hadron Energy & 0.0006 & 0.0006 \\
NC/CC Separation & 0.0011 & 0.0007 \\
& & \\
$\nu_{e}$ Flux & 0.003 & 0.0007 \\
\hline $\begin{array}{l}\text { Structure Functions } \\
m_{e}\end{array}$ & 0.0021 & 0.011 \\
\hline Total Error & 0.0025 & 0.0005 \\
\hline
\end{tabular}

Table 3

Significant Sources of Error in a $\sin ^{2} \theta_{W}$ Determination for E-770 and E-815. Several small sources have not been listed but are included in the total. The reduction in the $\nu_{\varepsilon}$ is achieved through the Sign-Selected Beam; the improvement in the $m_{c}$ error is obtained using $R^{-}$.

sis and are well-understood.[12] The total error in the Sign-Selected Beam will be approximately \pm 0.0024 or about $1 \%$. The E-815 errors, compared with the expected final errors in E-744/E770 , are shown in Table 3 .

A likely future upgrade to the detector is a device to measure the $\nu_{e}$ flux in the beam; with the $\nu_{e}$ component accurately determined, data from quadrupole triplet (non-sign selected) and the sign-selected beam can be jointly used. Future quadrupole triplet runs have the advantage, especially in the Main Injector era, of increasing the data sample by more than an order-ofmagnitude. Such a run could use the measured $\rho$ as input to improve the error on $\sin ^{2} \theta_{W}$ as well as provide high-statistics structure function measurements. The higher statistics could also be used to measure $m_{c}$, useful both in its own right and as an input to the electroweak measurement. With 15 million $\nu_{\mu}$ events, E-815 could determine $\sin ^{2} \theta_{W}$ to \pm 0.0012 , corresponding to $\Delta M_{W}= \pm 0.060 \mathrm{GeV} / \mathrm{c}^{2}$. 


\subsection{High Statistics Structure Function Determinations}

The nearly twenty million $\nu_{\mu}$ events at the Main Injector could be used for a number of precision QCD tests as well. The logarithmic derivative of $F_{3}$ vs. $Q^{2}$ is proportional to $\alpha_{S}$, and hence the slope can be used to determine $\Lambda_{\mathrm{QCD}}$. E-744/770 has determined $\Lambda_{\overline{\mathrm{MS}}}$ to \pm 28 (stat) \pm 41 (syst.) and the factor of ten additional statistics would make the statistical error negligible.[13] Improvements in the systematic error require better understanding of the relative calibration between the hadron shower energy, measured in scintillators, and the muon momentum, measured in a toroid. Studies of possible techniques are underway. Other tests, such as the GLS Sum Rule, would also benefit from the improved statistical power and systematic precision.[14] A factor of ten increase in dimuon statistics would provide both a precise determination of $m_{c}$ as an internal input for the experiment's determination of $\sin ^{2} \theta_{W}$ and as a check of NLO corrections to the charm cross-section.[15]

\section{Searches for Neutrino Oscillations Us- ing the Debuncher}

P-860 utilizes an unconventional neutrino beam from the Fermilab debuncher ring in order to study neutrino oscillations.[16] While the primary focus of the experiment is on $\nu_{e} \rightarrow \nu_{\tau}$ oscillation, the experiment is also sensitive to a wide range of parameters for $\nu_{e} \rightarrow \nu_{\tau}$ and, to a lesser extent, $\nu_{\mu} \rightarrow \nu_{\tau}$. Although the initial phase of the program would focus on a short baseline experiment, the addition of a second detector at some later time $5-10 \mathrm{~km}$ from the Debuncher would greatly expand the initial search and would be particularly interesting for a $\nu_{\mu} \rightarrow \nu_{\tau}$ oscillation study. The increased flux available when the new Main Injector is operational would also be a great advantage for this long baseline phase of the experiment.

The Debuncher is a strong focusing synchrotron which is used to debunch the antiprotons used in the Tevatron Collider. However, the Debuncher also accepts secondaries which originate at the antiproton production target: neg-

\begin{tabular}{|ccc|}
\hline$\nu_{\mu}(\pi$-decay & $\nu_{e}(\mu$-decay $)$ & $\bar{\nu}_{\mu}(\mu$-decay $)$ \\
\hline $4000 \mathrm{~K}$ & $140 \mathrm{~K}$ & $300 \mathrm{~K}$ \\
\hline
\end{tabular}

Table 4

Interaction Rates in the Proposed Experiment in a Near Detector.

\begin{tabular}{|l|rr|}
\hline & $3 \mathrm{GeV}$ & $9 \mathrm{GeV}$ \\
\hline $3 \mathrm{~km}$ & $10 \mathrm{~K}$ & $125 \mathrm{~K}$ \\
$10 \mathrm{~km}$ & $0.9 \mathrm{~K}$ & $11 \mathrm{~K}$ \\
\hline
\end{tabular}

Table 5

Number of charged-current $\nu_{\mu}$ events in a LongBaseline detector for $3 \mathrm{GeV}$ and $9 \mathrm{GeV}$ operation of the debuncher. A comparison of the two modes could be used to determine the nature of a signal.

ative pions, muons, and electrons are captured as well as the desired $\vec{p}$ 's. Within the first few turns, the majority of $\pi^{-}$decay to give $\mu^{-}$and $\bar{\nu}_{\mu}$. The muons which are trapped in the ring decay over many turns producting $\nu_{\mu}$ and $\bar{\nu}_{e}$. The Debuncher therefore naturally produces a timeseparated electron antineutrino beam. Its energy distribution is in the range of $3-9 \mathrm{GeV}$, so most of the beam is above threshold for producing $\tau$ leptons.

The rates from conventional collider operation are too low to be of use; hence the experiment requires a dedicated run in which positive pions are accumulated; this could occur during a Fermilab Fixed Target run.

The detector would consist of Iarocei tubes and scintillator in a toroidally magnetized iron spectrometer. The total mass in the $1.25 \mathrm{~m}$ radius, $183 \mathrm{ft}$ long apparatus is 1230 tons. Rates for a short and long-baseline detector in the envisaged beam are given in Tables 4 and 5 . The far detector rates are based on assuming $90 \%$ of the mass of the near detector has been moved to the far location. Since the statistics of the near detector are much higher, the effect on the errors are unimportant.

Oscillation Limits for the experiment are shown in Fig. 2. $\mathrm{P}-860$ is clearly capable of confirming 
or refuting the existence of the $17 \mathrm{keV}$ neutrino and measuring much of the same parameter space as the other oscillation experiments proposed at CERN and Fermilab.

\section{Tagged Neutrinos}

Were oscillations found in either P-860 or one of the other oscillation experiments, it would be difficult to precisely measure the oscillation parameters. Neither the neutrino energy nor its flight path $L$ are known for a given event, and precise statistical fits to the parameters would require far more events than would be available in the detectors.

One way to measure both the flight path and energy is to create a tagged neutrino beam from the semileptonic decay $K_{L} \rightarrow \pi e \nu_{e}$ or $K_{L} \rightarrow$ $\pi \mu \nu_{\mu}$. The experiment is conceptually simple.[17] A $K_{L}$ beam and the decay modes $K_{L} \rightarrow \pi \mu \nu_{\mu}$ and $K_{L} \rightarrow \pi e \nu_{\varepsilon}$ provide the neutrino flux. An upstream tagging spectrometer then identifies the hadron and lepton and reconstructs the $K_{L}$ decay; the lepton identification specifies the neutrino as $\nu_{e}$ or $\nu_{\mu}$ and distinguishes $\nu$ from $\bar{\nu}$ at the decay vertex. A neutrino detector modeled after an existing deep-inelastic scattering spectrometer (rates have been worked out for the CCFR apparatus) can be used to associate the $K_{L}$ with a neutrino interaction, measure the neutrino energy, and analyze outgoing muons. Monte Carlo studies show that $30 \mathrm{~K} \nu_{\mathrm{e}}$ and $20 \mathrm{~K} \nu_{\mu}$ could be obtained in two fixed target runs at the Tevatron.

The experiment will significantly improve existing oscillation limits, but it is especially sensitive to $\nu_{e} \rightarrow \nu_{\tau}$ oscillations. By searching for $\nu_{\tau} N \rightarrow \tau X, \tau \rightarrow \mu \nu \nu$ we may use the easyto-identify muon as a signal for oscillations. In conventional accelerator-based experiments in $\nu_{\mu}$ beams, we search for $\nu_{\mu} \rightarrow \nu_{\mathrm{e}}$ and $\nu_{\mu} \rightarrow \nu_{\tau}$, and (1) the absence of a muon or (2) the presence of an electron, signals oscillations. Either conventional method poses formidable systematic barriers to a conclusive discovery. The tagging experiment could search to $\sin ^{2} 2 \theta \approx 2 \times 10^{-3}$ at $90 \% \mathrm{CL}$ before backgrounds became significant, a factor of seventy better than existing limits. Constraining the pion and lepton to come from a $K_{L}$ de- cay provides a prediction of the neutrino impact point and energy, which can be compared to the measured values in the neutrino detector. The backgrounds at the neutrino vertex are well understood after years of deep-inelastic scattering experiments at the Tevatron.

Oscillations from $\nu_{\mu} \rightarrow \nu_{\tau}$ could be detected as well. By identifying the initial $\nu_{\mu}$, a prediction for the charged-current neutrino rate in the spectrometer could be checked against the observed charged-current rate. Since $82 \%$ of $\tau$ decays are muonless, $\nu_{\tau}$ interactions would appear as "neutral currents"; i.e., events with no muons. Errors include the fluctuations in the real neutral current rate and the knowledge of the branching fraction of $\tau$ into hadrons. A simulation indicates that a 1 kton detector would have $\approx 10 \mathrm{~K}$ events; hence the NC rate from $\nu_{\mu}$ would be about $3 \mathrm{~K}$ events with an error of \pm 55 , or $0.5 \%$. The $\Delta m^{2}$ reach is dependent on geometry but could go as low as approximately $1 \mathrm{eV}^{3}$. Hence if a signal is seen in one of the oscillation experiments a tagged neutrino experiment could verify the effect and measure the oscillation parameters over a wide range.

The upcoming generation of $K_{L}$ experiments at the Tevatron and the Main Injector could provide a tagging spectrometer far better than would be needed for these purposes.[18] A. first generation $(\approx 200$ ton) detector mounted downstream could prove the tagging concept works and discover or rule out the $17 \mathrm{keV}$ neutrino (assumed to be the $\nu_{\tau}$ ) at $\sin ^{2} 2 \theta>1 \times 10^{-3}$. By studying $\sigma\left(\nu_{e}\right) / \sigma\left(\nu_{\mu}\right)$ as a function of $E_{\nu}$, we could check for oscillations of $\nu_{a}$ into sterile neutrinos.

It is worth pointing out that a tagged neutrino line would provide a unique handle on a variety of fundamental questions. The ratio of $\nu_{\varepsilon}$ to $\nu_{\mu}$ cross-sections would be measured to better than $1 \%$, and a number of new electroweak tests could be performed.[19] In any case, either a tagged neutrino beam or P-860 would be the world's first copious, clean source of high-energy $\nu_{e}$. Either experiment would provide the first look at a new type of physics.

Fermilab is operated by Universities Research Association Inc. under Contract DEAC02-76CHO3000 with the United States De- 


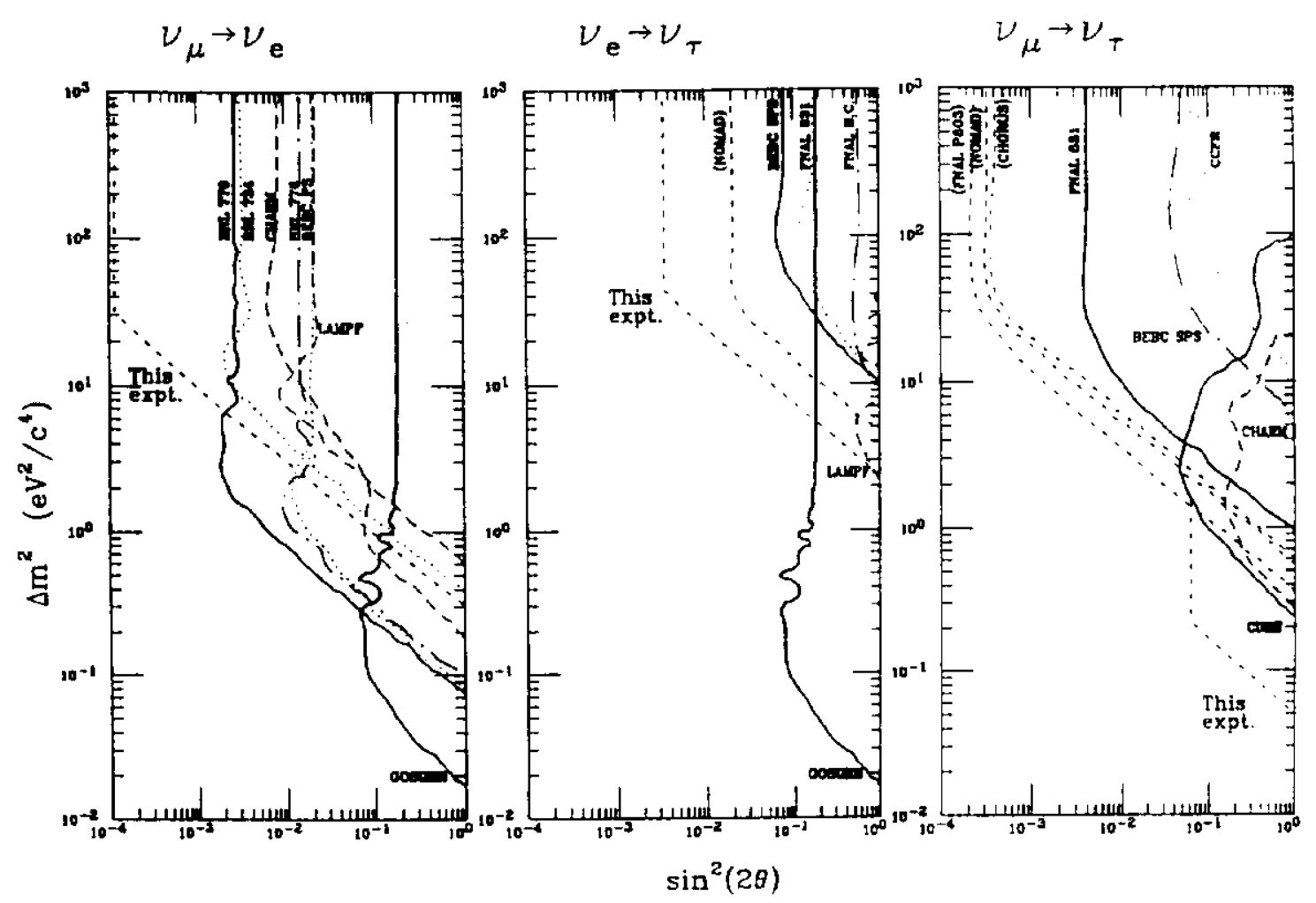

Figure 2. Oscillation Limits from P-860.

partment of Energy.

\section{REFERENCES}

1 J. Schneps, this Conference.

2 H.E. Fisk and F. Sciulli, Ann. Rev. Nucl. Sci., 32(1982) 499. The detector was instrumented with drift chambers for the Tevatron. See B.A. Schumm, Like-Sign Dimuon Production..., PhD Thesis, U. of Chicago, August 1988.

3 P-815 proposal, Precision Measurements of Neutrino Neutral Current Interactions Using a Sign-Selected Beam, and references therein.

4 A. Sirlin, Phys. Rev. D22 (1980) 971.

5 M. Veltman, Nucl. Phys. B123,(1977) 89.

6 C.H. Llewellyn Smith, Nucl. Phys. B228 (1983) 205.

7 E.A. Paschos and L. Wolfenstein, Phys. Rev. D7 (1973) 91.

8 P. Langacker, M. Luo, and A. Mann, Rev. Mod. Phys. 64 (1992) 87.

9 W.H. Smith, this Conference.

10 A. Blondel et al., Z. Phys. C45 (1990) 361.

11 S.R. Mishrs et al., Measurements of $\mathrm{Nu}$ cleon Structure Functions..., Nevis Preprint \#1459 (submitted to Phys. Rev. Lett)

12 M. Shaevits, this Conference.
13 P.Z. Quintas et al., A Measurement of $\Lambda_{M s}$ ..., Nevis Preprint \#1461, submitted to Phys. Rev. Lett.

14 W.C. Leung et al., A Measurement of the Gross-Llewellyn Smith Sum Rule from the CCFR $\approx F_{3}$ Structure Function, Nevis Preprint \#1460, submitted to Phys. Rev. Lett.

15 M. Aivasis, F. Olness, and Wu-Ki Tung, Phys. Rev. Lett. 65, (1990) 2339.

16 P-860, A Search for Neutrino Oscillations Using the Fermilab Debunchez and references therein.

17 A Proposal for a Neutrino Oscillation Experiment in a Tagged Neutrino Line, P-788, September 1988 and references therein.

18 Kaons at the Main Injector, Conceptual Design Report, Fermilab, 1992 (unpublished). Also see P-799 and P-832 at Fermilab.

19 R. Bernstein, ${ }_{A} A$ New Method of Determining $\sin ^{2} \theta_{W}$ in Deep-Inelastic $\nu_{\mu} N$ Scattering," Nucl.Phys. B13 (1990) 335. Also see "The $17 \mathrm{keV}$ Neutrino and Neutrino Tagging, ${ }^{n}$ FNAL Conf 92/062 (presented at Workshop on Long-Baseline Neutrino Oscillations, Batavia IL 17-20 November 1991). 